\title{
Research Article \\ Fixed Points, Maximal Elements and Equilibria of Generalized Games in Abstract Convex Spaces
}

\author{
Yan-Mei Du \\ Department of Mathematics, Tianjin Polytechnic University, Tianjin, Hedong 300387, China \\ Correspondence should be addressed to Yan-Mei Du, duyanmei@tjpu.edu.cn
}

Received 24 October 2012; Accepted 23 November 2012

Academic Editor: Yongfu Su

Copyright (C) 2012 Yan-Mei Du. This is an open access article distributed under the Creative Commons Attribution License, which permits unrestricted use, distribution, and reproduction in any medium, provided the original work is properly cited.

We firstly prove some new fixed point theorems for set-valued mappings in noncompact abstract convex space. Next, two existence theorems of maximal elements for class of $\mathcal{A}_{C, \theta}$ mapping and $A_{C, \theta}$-majorized mapping are obtained. As in applications, we establish new equilibria existence theorems for qualitative games and generalized games. Our theorems improve and generalize the most known results in recent literature.

\section{Introduction}

Since Borglin and Keiding [1] proved a new existence theorem for a compact generalized games (=abstract economy) with $K F$-majorized preference correspondences. Following their ideas, many authors studied the existence of equilibria for generalized games, for example; see Ding [2], Shen [3], Chowdhury et al. [4], Briec and Horvath [5], Ding and Wang [6], Kim et al. [7], Kim and Tan [8], Lin et al. [9], Lin and Liu [10], Du and Deng [11], and so forth. In the setting, convexity assumptions play a crucial role. Since Horvath $[12,13]$ introduced $H$ space by replacing convex hulls by contract subsets, many authors have put forward abstract convex spaces without linear structure, for example: G-convex space [14] and FC-space [15]. As a result, many authors established existence theorems of maximal elements and equilibria of generalized games with majorized correspondences in $H$-space, G-convex space, and FCspace, respectively. For example, see Hou [16], Ding [17], Ding and Xia [18], Yang and Deng [19], Ding and Feng [20], and others.

In 2006, Park [21] introduce the concept of an abstract convex space, which is a topological space without any convexity structure and linear structure. Moreover, abstract convex space include topological vector spaces, $H$-space, $G$-convex space, and FC-space as special cases (see Park [21-23]). Abstract convex space will be the framework of this paper. 
In this paper, we will introduce the new class of $\mathcal{A}_{C, \theta}$ mapping and $\mathcal{A}_{C, \theta}$-majorized mapping in abstract convex space. Some new fixed point theorems for set-valued mappings are proved under very weak coercive conditions. Next, two existence theorems of maximal elements for class of $\mathscr{A}_{C, \theta}$ mapping and $\mathscr{A}_{C, \theta}$-majorized mapping are obtained. As in applications, we establish new equilibria existence theorems for qualitative games and generalized games. Our results generalized and improve the corresponding results due to Ding and Feng [20], Ding and Wang [6], Park [22, 24], Yuan [25], Chowdhury et al. [4], Tan and Yuan [26], Borglin-Keiding [1], Yannelis [27], and so forth.

\section{Preliminaries}

Let $X$ be a nonempty subset of topological space $E$. We shall denote by $2^{X}$ the family of all subsets of $X$, by $\langle X\rangle$ the family of all nonempty finite subsets of $X$, by $\operatorname{int}_{E}(X)$, the interior of $X$ in $E$, and by $\mathrm{cl}_{E}(X)$ the closure of $X$ in $E$.

If $Y$ is a topological space and $T, S: X \rightarrow 2^{Y}$ are two mappings, for any $D \subset X$ and $y \in Y$, let $S(D)=\cup_{x \in D} S(x)$ and $S^{-}(y)=\{x \in X: y \in S(x)\}$. The dom $S$ denotes the domain of $S$, that is, $\operatorname{dom} S=\{x \in X: S(x) \neq \emptyset\}$, and $T \cap S: X \rightarrow 2^{Y}$ is a mapping defined by $(T \cap S)(x)=$ $T(x) \cap S(x)$ for each $x \in X$. The graph of $T$ is the set $\operatorname{Gr}(T)=\{(x, y) \in X \times Y: y \in T(x)\}$, and the mapping $\bar{T}: X \rightarrow 2^{Y}$ is defined by $\bar{T}(x)=\left\{y \in Y:(x, y) \in \mathrm{cl}_{X \times Y}(\operatorname{Gr}(T))\right\}$. The mapping cl $T: X \rightarrow 2^{Y}$ is defined by $(\operatorname{cl} T)(x)=\operatorname{cl}_{Y}(T(x))$ for each $x \in X$.

A subset $A$ of $E$ is said to be compactly open (resp., compactly closed) in $E$ if for each nonempty compact subset $C$ of $E, A \cap C$ is open (resp., closed) in $E$. Ding [28] define the compact interior and the compact closure of $A$ denoted by $\operatorname{cint}(A)$ and $\operatorname{ccl}(A)$ as

$$
\begin{aligned}
& \operatorname{cint}(A)=\bigcup\{B \subset E: B \subset A \text { and } B \text { is compactly open in } E\}, \\
& \operatorname{ccl}(A)=\bigcap\{B \subset E: A \subset B \text { and } B \text { is compactly closed in } E\} .
\end{aligned}
$$

It is easy to see that for any nonempty compact subset $K$ of $E$, we have $\operatorname{cint}(A) \cap K=\operatorname{int}_{K}(A \cap$ $K), \operatorname{ccl}(A) \cap K=\operatorname{cl}_{K}(A \cap K)$, and hence $\operatorname{cint}(A)$ (resp., $\left.\operatorname{ccl}(A)\right)$ is compactly open (resp., compactly closed) in $E$. By the definitions, a subset $A$ of $E$ is compactly open (resp., compactly closed) in $E$ if and only if $\operatorname{cint}(A)=A$ (resp., $\operatorname{ccl}(A)=A$ ).

Definition 2.1 (see $[21,22])$. An abstract convex space $(E, D ; \Gamma)$ consists of a topological space $E$, a nonempty set $D$, and a mapping $\Gamma:\langle D\rangle \rightarrow 2^{E}$ with nonempty values $\Gamma_{A}=\Gamma(A)$ for each $A \in\langle D\rangle$.

For any $D^{\prime} \subset D$, the $\Gamma$-convex hull of $D^{\prime}$ is denoted and defined by

$$
\operatorname{co}_{\Gamma} D^{\prime}=\cup\left\{\Gamma_{A} \mid A \in\left\langle D^{\prime}\right\rangle\right\} \subset E
$$

A subset $X$ of $E$ is called a $\Gamma$-convex subset of $(E, D ; \Gamma)$ relative to $D^{\prime}$ if for any $N \in$ $\left\langle D^{\prime}\right\rangle$, we have $\Gamma_{N} \subset X$, that is, $\operatorname{co}_{\Gamma} D^{\prime} \subset X$. Then, $\left(X, D^{\prime} ;\left.\Gamma\right|_{\left\langle D^{\prime}\right\rangle}\right)$ is called a $\Gamma$-convex subspace of $(E, D ; \Gamma)$.

When $D \subset E$, the space is denoted by $(E \supset D ; \Gamma)$. In such a case, a subset $X$ of $E$ is said to be $\Gamma$-convex if $\operatorname{co}_{\Gamma}(X \cap D) \subset X$; in other words, $X$ is $\Gamma$-convex relative to $D^{\prime}=X \cap D$. If $E=D$, let $(E ; \Gamma)=(E, E ; \Gamma)$. 
Definition 2.2 (see [22]). Let $(E, D ; \Gamma)$ be an abstract convex space. If a mapping $G: D \rightarrow 2^{E}$ satisfies

$$
\Gamma_{A} \subset G(A)=\bigcup_{y \in A} G(y) \quad \forall A \in\langle D\rangle,
$$

Then, $G$ is called a KKM mapping.

Definition 2.3 (see [22]). The partial KKM principle for an abstract convex space $(E, D ; \Gamma)$ is the statement that, for any closed-valued KKM mapping $G: D \rightarrow 2^{E}$, the family $\{G(y)\}_{y \in D}$ has the finite intersection property.

Definition 2.4. Let $X$ be a topological space, and $Y$ be a nonempty subset of an abstract convex space $(E ; \Gamma)$. Let $\theta: X \rightarrow Y$ be a single-valued mapping. Then, the mappings $\psi, \phi: X \rightarrow 2^{Y}$ are said to be an $\mathcal{A}_{C, \theta}$-pair if

(a) for each $x \in X, \theta(x) \notin \operatorname{co}_{\Gamma}(\phi(x)) \subset Y$ and $\psi(x) \subset \phi(x)$,

(b) the mapping $\psi^{-}: Y \rightarrow 2^{X}$ is compactly open valued on $Y$.

Definition 2.5. Let $X$ be a topological space, and $Y$ be a nonempty subset of an abstract convex space $(E ; \Gamma)$. Let $\theta: X \rightarrow Y$ be a single-valued mapping, and $P: X \rightarrow 2^{Y}$ be a set-valued mapping. Then,

(i) $P$ is said to be of class $\mathscr{A}_{C, \theta}$ if there exists an $\mathscr{A}_{C, \theta}$-pair such that

(a) $\operatorname{dom} P \subset \operatorname{dom} \psi$,

(b) for each $x \in X, P(x) \subset \phi(x)$.

(ii) $\left(\psi_{x}, \phi_{x} ; N_{x}\right)$ is said to be an $\mathcal{A}_{C, \theta}$-majorant of $P$ at $x \in X$ if $N_{x}$ is an open neighborhood of $x$ in $X$ and the mapping $\phi_{x}, \psi_{x}: X \rightarrow 2^{Y}$ such that

(a) for each $z \in N_{x}, P(z) \subset \phi_{x}(z), \theta(z) \notin \operatorname{co}_{\Gamma}\left(\phi_{x}(z)\right)$ and $\left\{z \in N_{x}: P(z) \neq \emptyset\right\} \subset$ $\left\{z \in N_{x}: \psi_{x}(z) \neq \emptyset\right\}$,

(b) for each $z \in X, \psi_{x}(z) \subset \phi_{x}(z)$ and $\operatorname{co}_{\Gamma}\left(\phi_{x}(z)\right) \subset Y$,

(c) the mapping $\psi_{x}^{-}: Y \rightarrow 2^{X}$ is compactly open valued on $Y$.

(iii) $P$ is said to be an $\mathscr{A}_{C, \theta}$-majorized mapping if for each $x \in X$ with $P(x) \neq \emptyset$, there exists an $\mathcal{A}_{C, \theta}$-majorant $\left(\psi_{x}, \phi_{x} ; N_{x}\right)$ of $P$ at $x$, and for any nonempty finite subset $A \in\langle\operatorname{dom} P\rangle$, the set $\left\{z \in \cap_{x \in A} N_{x}: P(z) \neq \emptyset\right\} \subset\left\{z \in \cap_{x \in A} N_{x}: \psi_{x}(z) \neq \emptyset\right\}$.

Remark 2.6. We note that our notions of the mapping $P$ being of class $\mathscr{A}_{C, \theta}$ (resp., $\mathscr{A}_{C, \theta^{-}}$ majorized) improve notions of mapping of class $\mathcal{L}_{\theta, F}^{*}$ (resp., $\mathcal{L}_{\theta, F}^{*}$-majorized), respectively introduced by Ding and Feng [20] from FC-space to abstract convex space, which in turn generalize the corresponding notions in Ding and Wang [6], Chowdhury et al. [4], Yuan [25], Ding et al. [29], and Ding [30].

In this paper, we shall deal mainly with either the case (I) $X=Y$, and $X$ is an abstract convex space, and $\theta=I_{X}$, which is the identity mapping on $X$ or the case (II) $X=\prod_{i \in I} X_{i}$, and $\theta=\pi_{i}: X \rightarrow X_{i}$ is the projection of $X$ onto $X_{i}$ and $X_{i}$ is an abstract convex space. In both cases (I) and (II), we shall write $\mathcal{A}_{C}$ in place of $\mathcal{A}_{C, \theta}$. 
Lemma 2.7 (see, $[24])$. Let $(E, D ; \Gamma)$ be an abstract convex space and $\left(X, D^{\prime} ; \Gamma^{\prime}\right)$ be a subspace. If $(E, D ; \Gamma)$ satisfies the partial KKM principle, then so does $\left(X, D^{\prime} ; \Gamma^{\prime}\right)$.

Lemma 2.8 (see, [22]). Let $(E, D ; \Gamma)$ be an abstract convex space satisfying the partial KKM principle, and $S: D \rightarrow 2^{E}$ be a mapping such that:

(i) for each $z \in X, S(z)$ is open,

(ii) $E=\bigcup_{z \in M} S(z)$ for some $M \in\langle D\rangle$.

then, there exists an $N \in\langle D\rangle$ such that $\Gamma_{N} \cap \bigcap_{z \in N} S(z) \neq \emptyset$.

\section{Fixed Point Theorems}

Theorem 3.1. Let $(E, D ; \Gamma)$ be an abstract convex space satisfying the partial KKM principle, and $K$ be a nonempty compact subset of $E$. Suppose that $F: E \rightarrow 2^{D}, G: E \rightarrow 2^{E}$ be mappings such that

(i) $F(x) \subset G(x)$ for each $x \in E$;

(ii) for each $y \in D, F^{-}(y)$ is compactly open in $E$ and for each $x \in K, F(x) \neq \emptyset$,

(iii) for each $N \in\langle D\rangle$, there exists a compact abstract convex subset $L_{N}$ of $E$ containing $N$ such that

$$
L_{N} \backslash K \subset \bigcup\left\{\operatorname{cint}\left(G^{-}(y)\right): y \in L_{N}\right\}
$$

Then, there exists a point $\widehat{x} \in E$, such that $\widehat{x} \in \mathrm{CO}_{\Gamma}(G(\widehat{x}))$.

Proof. Since for each $x \in K, F(x) \neq \emptyset$, then $K \subset \cup\left\{F^{-}(y): y \in D\right\}$. By (i) and (ii), then $K \subset$ $\left\{\operatorname{cint}\left(G^{-}(y)\right): y \in D\right\}$. Since $K$ is a nonempty compact subset of $E$, there exists a finite set $N \in\langle D\rangle$ such that

$$
K \subset \bigcup\left\{\operatorname{cint}\left(G^{-}(y)\right): y \in N\right\} .
$$

By (iii), there exists a compact abstract convex subset $L_{N}$ of $E$ containing $N$ such that

$$
L_{N} \backslash K \subset \bigcup\left\{\operatorname{cint}\left(G^{-}(y)\right): y \in L_{N}\right\} .
$$

By (3.2) and $N \subset L_{N}$, then

$$
L_{N} \cap K \subset \bigcup\left\{\operatorname{cint}\left(G^{-}(y)\right): y \in N\right\} \subset \bigcup\left\{\operatorname{cint}\left(G^{-}(y)\right): y \in L_{N}\right\} .
$$

By (3.3) and (3.4), we have

$$
L_{N} \subset \bigcup\left\{\operatorname{cint}\left(G^{-}(y)\right): y \in L_{N}\right\} .
$$

Since $L_{N}$ is compact, there exists a finite set $B=\left\{z_{1}, z_{2}, \ldots, z_{m}\right\} \in\left\langle L_{N}\right\rangle$, such that

$$
L_{N}=\bigcup_{i=1}^{m} \operatorname{cint}\left(G^{-}\left(z_{i}\right)\right) \cap L_{N}=\bigcup_{i=1}^{m} \operatorname{int}_{L_{N}}\left(G^{-}\left(z_{i}\right) \cap L_{N}\right) .
$$


Let $D^{\prime}=L_{N} \cap D$ and define $\Gamma^{\prime}:\left\langle D^{\prime}\right\rangle \rightarrow 2^{L_{N}}$ by $\Gamma_{A}^{\prime}=\Gamma_{A} \cap L_{N}$ for each $A \in\left\langle D^{\prime}\right\rangle$, then $\left(L_{N}\right.$, $\left.D^{\prime} ; \Gamma^{\prime}\right)$ is an abstract convex space. Since $(E, D ; \Gamma)$ satisfies the partial KKM principle, so does $\left(L_{N}, D^{\prime} ; \Gamma^{\prime}\right)$ by Lemma 2.7 .

Define $S: L_{N} \rightarrow 2^{L_{N}}$ by $S(z)=\operatorname{cint}\left(G^{-}(z)\right) \cap L_{N}$ for each $z \in L_{N}$. It is easy to prove that the all the hypotheses of Lemma 2.8 are satisfied. By Lemma 2.8, there exists a finite set $M \in\left\langle L_{N}\right\rangle$ such that $\Gamma_{M}^{\prime} \cap \bigcap_{z \in M} S(z) \neq \emptyset$. Let $\hat{x} \in \Gamma_{M}^{\prime} \cap \bigcap_{z \in M} S(z)$, then for each $z \in M, \widehat{x} \in S(z)=\operatorname{cint}\left(G^{-}(z)\right) \cap L_{N} \subset G^{-}(z)$, that is $M \subset G(\widehat{x})$ and $\Gamma_{M} \subset \operatorname{co}_{\Gamma}(G(\widehat{x}))$. Since $\widehat{x} \in \Gamma_{M}^{\prime}=\Gamma_{M} \cap L_{N} \subset \Gamma_{M}$, thus $\widehat{x} \in \operatorname{co}_{\Gamma}(G(\widehat{x}))$. This completes the proof.

Remark 3.2. Theorem 3.1 generalizes Theorem 3.1 of Ding and Feng [20] and Theorem 3.1 of Ding and Wang [6] from FC-space to abstract convex space, and the coercive condition (iii) in Theorem 2.1 is weaker than the condition (3) in Theorem 3.1 of Ding and Wang [6].

Corollary 3.3. Let $(E, D ; \Gamma)$ be an abstract convex space satisfying the partial KKM principle, and $K$ be a nonempty compact subset of $E$. Suppose that $F: E \rightarrow 2^{D}, G: E \rightarrow 2^{E}$ be mappings such that

(i) $F(x) \subset G(x)$ for each $x \in E$,

(ii) for each $y \in D, F^{-}(y)$ is compactly open in $E$ and for each $x \in K, F(x) \neq \emptyset$,

(iii) for each $N \in\langle D\rangle$, there exists a compact abstract convex subset $L_{N}$ of $E$ containing $N$ such that for each $x \in L_{N} \backslash K$, there exists a point $\bar{y} \in L_{N}$ such that $x \notin \mathrm{cl}_{X}\left[X \backslash G^{-}(\bar{y})\right]$.

Then, there exists a point $\widehat{x} \in E$, such that $\widehat{x} \in \mathrm{co}_{\Gamma}(G(\widehat{x}))$.

Proof. By (iii), for each $N \in\langle D\rangle$, there exists a compact abstract convex subset $L_{N}$ of $E$ containing $N$ such that for each $x \in L_{N} \backslash K$, there exists $\bar{y} \in L_{N}$ such that $x \notin \mathrm{cl}_{X}\left[X \backslash G^{-}(\bar{y})\right]$, then $x \in\left(\operatorname{cl}_{X}\left[X \backslash G^{-}(\bar{y})\right]\right)^{c}=\operatorname{int}\left(G^{-}(\bar{y})\right)$, thus $x \in \cup\left\{\operatorname{int}\left(G^{-}(y)\right): y \in L_{N}\right\} \subset\left\{\operatorname{cint}\left(G^{-}(y)\right): y \in L_{N}\right\}$, that is $L_{N} \backslash K \subset\left\{\operatorname{cint}\left(G^{-}(y)\right): y \in L_{N}\right\}$. Hence, all the hypotheses of Theorem 3.1 are satisfied. By Theorem 3.1, there exists a point $\widehat{x} \in E$, such that $\widehat{x} \in \mathrm{Co}_{\Gamma}(G(\widehat{x}))$. This completes the proof.

Remark 3.4. Corollary 3.3 generalizes Theorem 3.2 of Ding and Feng [20] from FC-space to abstract convex space. Moreover, Corollary 3.3 improves the corresponding result of Park $[22,24]$.

\section{Existence of Maximal Elements}

Let $X$ be a topological space, and $P: X \rightarrow 2^{X}$ be a mapping. A point $\widehat{x} \in X$ is called a maximal element of $T$ if $T(\widehat{x})=\emptyset$.

In the section, We firstly prove a selection theorem for $\mathcal{A}_{C}$-majorized mapping. Next, we will establish some new existence theorems of maximal elements for class $\mathscr{A}_{C}$ mapping and $\mathscr{A}_{C}$-majorized mapping defined on noncompact abstract convex space.

Lemma 4.1. Let $X$ be a regular topological space, and $Y$ be a nonempty subset of an abstract convex space $(E ; \Gamma)$. Let $\theta: X \rightarrow E$, and $P: X \rightarrow 2^{Y}$ be an $A_{C, \theta}$-majorized mapping. If each open subset of $X$ containing the set $B=\operatorname{dom} P$ is paracompact, then there exists a $A_{C, \theta}$-pair $\psi, \phi: X \rightarrow 2^{Y}$ such that $P(x) \subset \phi(x)$ for each $x \in X$ and $\operatorname{dom} P \subset \operatorname{dom} \psi$. 
Proof. Since $P$ is an $\mathcal{A}_{C, \theta}$-majorized mapping, for each $x \in B$, let $N_{x}$ be an open neighborhood of $x$ in $X$, and $\psi_{x}, \phi_{x}: X \rightarrow 2^{Y}$ be mappings such that

(1) for each $z \in N_{x}, P(z) \subset \phi_{x}(z), \theta(z) \notin \operatorname{co}_{\Gamma}\left(\phi_{x}(z)\right)$ and $\left\{z \in N_{x}: P(z) \neq \emptyset\right\} \subset\{z \in$ $\left.N_{x}: \psi_{x}(z) \neq \emptyset\right\}$,

(2) for each $z \in X, \psi_{x}(z) \subset \phi_{x}(z)$ and $\operatorname{co}_{\Gamma}\left(\phi_{x}(z)\right) \subset Y$,

(3) the mapping $\psi_{x}^{-}: Y \rightarrow 2^{X}$ is compactly open valued on $Y$,

(4) for each $A \in\langle\operatorname{dom} P\rangle,\left\{z \in \cap_{x \in A} N_{x}: P(z) \neq \emptyset\right\} \subset\left\{z \in \cap_{x \in A} N_{x}: \cap_{x \in A} \psi_{x}(z) \neq \emptyset\right\}$.

Since $X$ is regular, for each $x \in B$, there exists an open neighborhood $G_{x}$ of $x$ in $X$ such that $\operatorname{cl}_{X} G_{x} \subset N_{x}$. Let $G=\cup_{x \in B} G_{x}$, then $G$ is an open subset of $X$ containing $B$, so that $G$ is paracompact by assumption. By Theorem VIII.1.4 of Dugundji [31], the open covering $\left\{G_{x}\right\}$ of $G$ has an open precise neighborhood finite refinement $G_{x}^{\prime}$. Given any $x \in B$, we define the mappings $\psi_{x}^{\prime}, \phi_{x}^{\prime}: G \rightarrow 2^{Y}$ by

$$
\begin{aligned}
& \psi_{x}^{\prime}(z)= \begin{cases}\psi_{x}(z) & \text { if } z \in G \cap \mathrm{cl}_{X} G_{x}^{\prime} \\
Y & \text { if } z \in G \backslash \mathrm{cl}_{X} G_{x}^{\prime}\end{cases} \\
& \phi_{x}^{\prime}(z)= \begin{cases}\phi_{x}(z) & \text { if } z \in G \cap \mathrm{cl}_{X} G_{x}^{\prime} \\
Y & \text { if } z \in G \backslash \mathrm{cl}_{X} G_{x}^{\prime}\end{cases}
\end{aligned}
$$

then we have

(i) by (2), for each $z \in G, \psi_{x}^{\prime}(z) \subset \phi_{x}^{\prime}(z)$,

(ii) by (1), dom $P \subset \operatorname{dom} \psi_{x}^{\prime}$, and $P(z) \subset \phi_{x}^{\prime}(z)$ for each $z \in G$,

(iii) for each $y \in Y$, the set

$$
\begin{aligned}
\left(\psi_{x}^{\prime}\right)^{-}(y) & =\left\{z \in G \cap \mathrm{cl}_{X} G_{x}^{\prime}: y \in \psi_{x}(z)\right\} \cup\left\{z \in G \backslash \mathrm{cl}_{X} G_{x}^{\prime}: y \in Y\right\} \\
& =\left[\left(G \cap \mathrm{cl}_{X} G_{x}^{\prime}\right) \cap \psi_{x}^{-}(y)\right] \cup\left(G \backslash \mathrm{cl}_{X} G_{x}^{\prime}\right) \\
& =\left[G \cap \psi_{x}^{-}(y)\right] \cup\left(G \backslash \mathrm{cl}_{X} G_{x}^{\prime}\right) .
\end{aligned}
$$

It follows that for each nonempty compact subset $C$ of $X$, the set

$$
\left(\psi_{x}^{\prime}\right)^{-}(y) \cap C=\left[G \cap \psi_{x}^{-}(y) \cap C\right] \cup\left[\left(G \backslash \mathrm{cl}_{X} G_{x}^{\prime}\right) \cap C\right]
$$

is open in $C$ by (3). Thus, the mapping $\left(\psi_{x}^{\prime}\right)^{-}: Y \rightarrow 2^{G}$ is compactly open valued on $Y$. Now, define $\psi, \phi: X \rightarrow 2^{\gamma}$ by

$$
\begin{gathered}
\psi(z)= \begin{cases}\bigcap_{x \in B} \psi_{x}^{\prime}(z) & \text { if } z \in G, \\
\emptyset & \text { if } z \in X \backslash G,\end{cases} \\
\phi(z)= \begin{cases}\cap \phi_{x \in B}^{\prime}(z) & \text { if } z \in G, \\
\emptyset & \text { if } z \in X \backslash G .\end{cases}
\end{gathered}
$$


(a) For each $z \in X$, by (i), $\psi(z) \subset \phi(z)$ and $\operatorname{co}_{\Gamma}(\phi(z)) \subset Y$. If $z \in X \backslash G$, then $\phi(z)=\emptyset$, so that $\theta(z) \notin \operatorname{co}_{\Gamma}(\phi(z))$; if $z \in G$, then $z \in G \cap \operatorname{cl}_{X} G_{x}^{\prime}$ for some $x \in B$, so that $\phi_{x}^{\prime}(z)=\phi_{x}(z)$, and hence $\phi(z) \subset \phi_{x}(z)$, by (1), we have $\theta(z) \notin \operatorname{co}_{\Gamma}(\phi(z))$. Therefore, $\theta(z) \notin \operatorname{co}_{\Gamma}(\phi(z))$ for all $z \in X$.

(b) Now, we show that the mapping $\psi^{-}: Y \rightarrow 2^{X}$ is compactly open valued on $Y$. Indeed, let $y \in Y$ be such that $\psi^{-}(y) \neq \emptyset$ and $C$ be a nonempty compact subset of $X$. Given a point $u$,

$$
u \in \psi^{-}(y) \cap C=\{z \in X: y \in \psi(z)\} \cap C=\{z \in G: y \in \psi(z)\} \cap C
$$

Since $\left\{G_{x}^{\prime}\right\}$ is a neighborhood finite refinement, there exists an open neighborhood $M_{u}$ of $u$ in $G$ such that $\left\{x \in B: M_{u} \cap G_{x}^{\prime} \neq \emptyset\right\}=\left\{x_{1}, x_{2}, \ldots, x_{n}\right\}$. Note that for each $x \in B$ with $x \notin\left\{x_{1}, x_{2}, \ldots, x_{n}\right\}, \emptyset=M_{u} \cap G_{x}^{\prime}=M_{u} \cap \operatorname{cl}_{X} G_{x}^{\prime}$, so that $\psi_{x}^{\prime}(z)=Y$ for $z \in M_{u}$. Then, we have $\psi(z)=\cap_{x \in B} \psi_{x}^{\prime}(z)=\cap_{i=1}^{n} \psi_{x_{i}}^{\prime}(z)$ for all $z \in M_{u}$. It follows that

$$
\begin{aligned}
\psi^{-}(y) & =\{z \in X: y \in \psi(z)\}=\left\{z \in G: y \in \bigcap_{x \in B} \psi_{x}^{\prime}(z)\right\} \\
& \supset\left\{z \in M_{u}: y \in \bigcap_{x \in B} \psi_{x}^{\prime}(z)\right\} \\
& =\left\{z \in M_{u}: y \in \bigcap_{i=1}^{n} \psi_{x_{i}}^{\prime}(z)\right\} \\
& =M_{u} \cap\left\{z \in G: y \in \bigcap_{i=1}^{n} \psi_{x_{i}}^{\prime}(z)\right\} \\
& =M_{u} \cap\left[\bigcap_{i=1}^{n}\left(\psi_{x_{i}}^{\prime}\right)^{-}(y)\right] .
\end{aligned}
$$

Since $\left(\psi_{x_{i}}^{\prime}\right)^{-}(y)$ is compactly open in $X$ by (iii), then $M_{u}^{\prime}=M_{u} \cap\left[\cap_{i=1}^{n}\left(\psi_{x_{i}}^{\prime}\right)^{-}(y)\right] \cap C$ is an open neighborhood of $u$ in $C$ such that $M_{u}^{\prime} \subset \psi^{-}(y) \cap C$. This shows that $\psi^{-}: Y \rightarrow 2^{X}$ is compactly open valued on $Y$.

By (a) and (b), thus $(\psi, \phi)$ is an $\mathscr{A}_{C, \theta}$-pair.

Next, we claim that $\operatorname{dom} P \subset \operatorname{dom} \psi$, indeed, for each $w \in \operatorname{dom} P$, we must have $w \in G$. Since $\left\{G_{x}^{\prime}\right\}$ is neighborhood finite, then the set $\left\{x \in B: w \in \operatorname{cl}_{X} G_{x}^{\prime}\right\}=\left\{x_{1}^{\prime}, x_{2}^{\prime}, \ldots, x_{m}^{\prime}\right\}$. If $x \notin$ $\left\{x_{1}^{\prime}, x_{2}^{\prime}, \ldots, x_{m}^{\prime}\right\}, w \in G \backslash \mathrm{cl}_{X} G_{x}^{\prime}$, and $\psi_{x}^{\prime}(w)=Y$, thus we have $\psi(w)=\cap_{x \in B} \psi_{x}^{\prime}(w)=\cap_{i=1}^{m} \psi_{x_{i}^{\prime}}^{\prime}(w)$. Since $w \in \cap_{i=1}^{m} \mathrm{cl}_{X} G_{x_{i}^{\prime}}^{\prime} \subset \cap_{i=1}^{m} N_{x_{i}^{\prime}}$, by $(4), \psi(w) \neq \emptyset$. Hence, $\operatorname{dom} P \subset \operatorname{dom} \psi$.

Finally, we prove that $P(z) \subset \phi(z)$ for each $z \in X$. Indeed, let $z \in X$ with $P(z) \neq \emptyset$, then $z \in G$. For each $x \in B$, if $z \in G \backslash \mathrm{cl}_{X} G_{x}^{\prime}$, then $\phi_{x}^{\prime}(z)=Y$, and so $P(z) \subset \phi_{x}^{\prime}(z)$, and if $z \in$ $G \cap \mathrm{cl}_{X} G_{x}^{\prime}$, we have $z \in \operatorname{cl}_{X} G_{x}^{\prime} \subset \operatorname{cl}_{X} G_{x} \subset N_{x}$, so that by (1), $P(z) \subset \phi_{x}(z) \subset \phi_{x}^{\prime}(z)$. It follows that $P(z) \subset \phi_{x}^{\prime}(z)$ for all $x \in B$ so that $P(z) \subset \cap_{x \in B} \phi_{x}^{\prime}(z)=\phi(z)$. This completes the proof.

Remark 4.2. Lemma 4.1 generalizes Lemma 4.1 of Ding and Feng [20], Lemma 5.1 of Ding and Wang [15], Theorem 3.1 of Yuan [25], Lemma 3.1 of Chowdhury et al. [4], and Lemma 3.1 of Tan and Yuan [26]. 
Theorem 4.3. Let $(X ; \Gamma)$ be an abstract convex space satisfying the partial KKM principle, and let $K$ be a nonempty compact subset of $X$. Suppose that the mapping $P: X \rightarrow 2^{X}$ is of class $\mathcal{A}_{C}$ and satisfy

(i) for each $N \in\langle X\rangle$, there exists a compact abstract convex subset $L_{N}$ of $X$ containing $N$ such that

$$
L_{N} \backslash K \subset \bigcup\left\{\operatorname{cint}\left(P^{-}(y)\right): y \in L_{N}\right\}
$$

Then, there exists a point $\widehat{x} \in K$ such that $P(\widehat{x})=\emptyset$.

Proof. Since $P$ is of class $\mathscr{A}_{C}$, then there exists an $\mathscr{A}_{C}$-pair $(\phi, \psi)$ such that

(a) $\operatorname{dom} P \subset \operatorname{dom} \psi, P(x) \subset \phi(x)$ for each $x \in X$,

(b) for each $x \in X, x \notin \operatorname{co}_{\Gamma}(\phi(x)) \subset X$ and $\psi(x) \subset \phi(x)$,

(c) the mapping $\psi^{-}: X \rightarrow 2^{X}$ is compactly open valued on $X$.

Suppose that for each $x \in K, P(x) \neq \emptyset$. By (a), $\psi(x) \neq \emptyset$ for each $x \in K$. By (i) and (a), for each $N \in\langle X\rangle$, there exists a compact abstract convex subset $L_{N}$ of $X$ containing $N$ such that $L_{N} \backslash K \subset \cup\left\{\operatorname{cint}\left(P^{-}(y)\right): y \in L_{N}\right\} \subset \cup\left\{\operatorname{cint}\left(\phi^{-}(y)\right): y \in L_{N}\right\}$. Therefore, $\psi$ and $\phi$ satisfy all the hypotheses of Theorem 3.1. By Theorem 3.1, there exists a point $\bar{x} \in K$ such that $\bar{x} \in \mathrm{CO}_{\Gamma}(\phi(\bar{x}))$. Which contradicts with condition (b). Hence, there exists a point $\widehat{x} \in K$ such that $P(\widehat{x})=\emptyset$. This completes the proof.

Remark 4.4. Theorem 4.3 generalizes most existence theorems of maximal elements in the literature, for example; see Theorem 4.1 of Ding and Feng [20], Theorem 3.2 of Yuan [25], Theorem 3.1 of Chowdhury et al. [4], Theorem 3.2 of Tan and Yuan [26], Theorem 5.2 of Ding and Wang [6], and so on.

As an application of Lemma 4.1 and Theorem 4.3, we have the following existence theorem maximal elements.

Theorem 4.5. Let $(X ; \Gamma)$ be a paracompact abstract convex space satisfying the partial KKM principle, and let $K$ be a nonempty compact subset of $X$. Let $P: X \rightarrow 2^{X}$ be an $A_{C}$ majorized mapping and satisfy

(i) for each $N \in\langle X\rangle$, there exists a compact abstract convex subset $L_{N}$ of $X$ containing $N$ such that

$$
L_{N} \backslash K \subset \bigcup\left\{\operatorname{cint}\left(P^{-}(y)\right): y \in L_{N}\right\}
$$

Then, there exists a point $\widehat{x} \in K$ such that $P(\widehat{x})=\emptyset$.

Proof. Suppose that $P(x) \neq \emptyset$ for all $x \in X$, then $\operatorname{dom} P=X$ is paracompact. By Lemma $4.1 P$ is of class $A_{C}$. Therefore, all the hypotheses of Theorem 4.3 are satisfied. By Theorem 4.3, there exists a point $\widehat{x} \in K$ such that $P(\widehat{x})=\emptyset$. Which is a contradiction. Thus, there exists a point $\widehat{x} \in X$ such that $P(\widehat{x})=\emptyset$. By the assumptions, $\widehat{x}$ must in $K$. This completes the proof.

Remark 4.6. Theorem 4.5 generalizes Theorem 5.3 of Ding and Wang [6], Theorem 3.2 of Chowdhury et al. [4], Theorem 3.3 of Tan and Yuan [26], Corollary 1 of Borglin-Keiding [1], and Theorem 2 of Yannelis [27]. 


\section{Existence of Equilibrium of Points}

Let $I$ be a (finite or infinite) set of players. For each $i \in I$, let its strategy set $X$ and $Y_{i}(i \in I)$ be nonempty set, and let $Y=\prod_{i \in I} Y_{i} . P_{i}: X \rightarrow 2^{Y_{i}}$ be the preference correspondence of $i$-th player. The collection $\Lambda=\left(X ; Y_{i} ; P_{i}\right)_{i \in I}$ will be called a qualitative game. A point $\widehat{x} \in X$ is said to be an equilibrium of the qualitative game, if $P_{i}(\widehat{x})=\emptyset$ for each $i \in I$.

A generalized game is a quintuple family $\Lambda=\left(X ; Y_{i} ; A_{i} ; B_{i} ; P_{i} ; \theta_{i}\right)_{i \in I}$, where $\mathrm{X}$ is a nonempty set, $I$ is a (finite or infinite) set of players such that for each $i \in I, Y_{i}$ is a nonempty set and $Y=\prod_{i \in I} Y_{i} . A_{i}, B_{i}: X \rightarrow 2^{Y_{i}}, \theta_{i}: X \rightarrow Y_{i}$ are the constraint correspondences, and $P_{i}: X \rightarrow 2^{Y_{i}}$ is the preference correspondence. An equilibrium of the generalized game $\Lambda$ is a point $\widehat{x} \in X$ such that for each $i \in I, \theta_{i}(\widehat{x}) \in \bar{B}_{i}(\widehat{x})$ and $A_{i}(\widehat{x}) \cap P_{i}(\widehat{x})=\emptyset$. If $\theta_{i}=\pi_{i}: X \rightarrow X_{i}$ is the projection of $X$ onto $X_{i}$, then our definition of an equilibrium point coincides with the standard definition given by Chowdhury et al. [4], and if, in addition, $A_{i}=B_{i}$ for each $i \in$ $I$, our definition of an equilibrium point generalizes the standard definition, for example, Borglin and Keiding [1] and Gale and Mas-Colell [32].

Lemma 5.1 (see [33]). Let $\left\{\left(X_{i}, D_{i} ; \Gamma_{i}\right)\right\}_{i \in I}$ be a family of abstract convex spaces. Let $X:=\prod_{i \in I} X_{i}$ be equipped with the product topology, and let $D:=\prod_{i \in I} D_{i}$. For each $i \in I$, let $\pi_{i}: D \rightarrow D_{i}$ be the projection. For each $A \in\langle D\rangle$, define $\Gamma(A):=\prod_{i \in I} \Gamma_{i}\left(\pi_{i}(A)\right)$. Then, $(X, D ; \Gamma)$ is an abstract convex space.

As an application of Theorem 4.3, we prove the following existence theorem of equilibrium points for one person game in abstract convex space.

Theorem 5.2. Let $(X ; \Gamma)$ be an abstract convex space satisfying the partial KKM principle and $K$ be a closed and compact subset of $X$, and let $K=\operatorname{co}_{\Gamma} K$. Suppose that the mappings $A, B$, and $P: X \rightarrow 2^{X}$ satisfy

(i) for each $x \in X, \mathrm{c}_{\Gamma}(\mathrm{A}(\mathrm{x})) \subset \overline{\mathrm{B}}(\mathrm{x})$ and $A(x) \cap P(x) \subset K$,

(ii) the mapping $A^{-}: X \rightarrow 2^{X}$ is compactly open valued on $X$,

(iii) the mapping $A \cap P: X \rightarrow 2^{X}$ is of class $A_{C}$ and $A(x) \neq \emptyset$ for each $x \in K$,

(iv) for each $N \in\langle X\rangle$, there exists a compact abstract convex subset $L_{N}$ of $X$ containing $N$ such that

$$
L_{N} \backslash K \subset \bigcup\left\{\operatorname{cint}\left((A \cap P)^{-}(y)\right): y \in L_{N}\right\}
$$

Then, there exists a point $\widehat{x} \in K$ such that $\widehat{x} \in \bar{B}(\widehat{x})$ and $A(\widehat{x}) \cap P(\widehat{x})=\emptyset$.

Proof. Let $W=\{x \in X: x \notin \bar{B}(x)\}$, then $W$ is open in $X$. Define $Q: X \rightarrow 2^{X}$ by

$$
Q(x)= \begin{cases}K & \text { if } x \in X \backslash K, \\ A(x) & \text { if } x \in K \cap W, \\ A(x) \cap P(x) & \text { if } x \in K \backslash W .\end{cases}
$$


By (iii), $A \cap P$ is of class $\mathcal{A}_{C}$, then there exist two mappings $\psi, \phi: X \rightarrow 2^{X}$ such that

(a) for each $x \in X, A(x) \cap P(x) \subset \phi(x)$ and $\operatorname{dom}(A \cap P) \subset \operatorname{dom} \psi$,

(b) for each $x \in X, x \notin \operatorname{co}_{\Gamma}(\phi(x))$ and $\psi(x) \subset \phi(x)$,

(c) the mapping $\psi^{-}: X \rightarrow 2^{X}$ is compactly open valued on $X$.

Define $\Psi, \Phi: X \rightarrow 2^{X}$ by

$$
\begin{gathered}
\Psi(x)= \begin{cases}K & \text { if } x \in X \backslash K, \\
A(x) & \text { if } x \in K \cap W, \\
\psi(x) & \text { if } x \in K \backslash W,\end{cases} \\
\Phi(x)= \begin{cases}K & \text { if } x \in X \backslash K, \\
A(x) & \text { if } x \in K \cap W, \\
\phi(x) & \text { if } x \in K \backslash W .\end{cases}
\end{gathered}
$$

Then, we have

(a') for each $x \in X, Q(x) \subset \Phi(x)$ by (a) and $\operatorname{dom} Q \subset \operatorname{dom} \Psi$,

$\left(\mathrm{b}^{\prime}\right)$ for each $x \in X, \Psi(x) \subset \Phi(x)$ by (b), if $x \in X \backslash K, x \notin K=\operatorname{co}_{\Gamma} K=\operatorname{co}_{\Gamma} \Phi(x)$ and if $x \in K \cap W, x \notin \bar{B}(x)$ by (i), and $x \notin \operatorname{co}_{\Gamma}(A(x))=\operatorname{co}_{\Gamma}(\Phi(x))$ and if $x \in K \backslash W$, $x \notin \operatorname{co}_{\Gamma}(\phi(x))=\operatorname{co}_{\Gamma}(\Phi(x))$ by (b), thus $x \notin \operatorname{co}_{\Gamma}(\Phi(x))$ for each $x \in X$,

$\left(c^{\prime}\right)$ for each $y \in X$, then it is easy to verify that the set

$$
\Psi^{-}(y)=\{x \in X: y \in \Psi(x)\}=(X \backslash K) \cup\left[A^{-}(y) \cap K \cap W\right] \cup\left[\Psi^{-}(y) \cap(K \backslash W)\right]
$$

is compactly open in $X$ by (ii) and (c). This shows that $Q$ is of class $\mathscr{A}_{C}$. By the definition of $Q$, condition (i) and (iv), for each $N \in\langle X\rangle$, there exists a compact abstract convex subset $L_{N}$ of $X$ containing $N$ such that

$$
L_{N} \backslash K \subset \bigcup\left\{\operatorname{cint}\left((A \cap P)^{-}(y)\right): y \in L_{N}\right\} \subset \bigcup\left\{\operatorname{cint}\left(Q^{-}(y)\right): y \in L_{N}\right\}
$$

Hence, all the hypotheses of Theorem 4.3 are satisfied. By Theorem 4.3 , there exists a point $\hat{x} \in K$ such that $Q(\hat{x})=\emptyset$. By the definition of $Q$ and condition (iii), $\hat{x} \in K \backslash W \subset K$, that is, $\widehat{x} \in \bar{B}(\widehat{x})$ and $A(\widehat{x}) \cap P(\widehat{x})=\emptyset$. This completes the proof.

Remark 5.3. Theorem 5.2 improves and generalizes Theorem 5.1 of Ding and Feng [20], Theorem 6.1 of Ding and Wang [6], Theorem 4.1 of Yuan [25], Theorem 4.1 of Chowdhury et al. [4], and Theorem 4.1 of Tan and Yuan [26].

As another application of Theorem 4.5, we can obtain the following existence of equilibria for qualitative games. 
Theorem 5.4. Let $\Lambda=\left(X ; X_{i} ; P_{i}\right)_{i \in I}$ be a qualitative game, For each $i \in I$, suppose that the following conditions are satisfied

(i) $\left(X_{i} ; \Gamma_{i}\right)_{i \in I}$ is a family of paracompact abstract convex space such that $(X ; \Gamma)$ satisfies the partial KKM principle, and $K$ is a nonempty compact subset of $X$,

(ii) $P_{i}: X \rightarrow 2^{X_{i}}$ is an $\mathbb{A}_{C}$-majorized mapping,

(iii) $W_{i}=\left\{x \in X: P_{i}(x) \neq \emptyset\right\}$ is open in $X$,

(iv) for each $N \in\langle X\rangle$, there exists a compact abstract convex subset $L_{N}$ of $X$ containing $N$ such that

$$
L_{N} \backslash K \subset \bigcup\left\{\operatorname{cint}\left(P_{i}^{-}\left(\pi_{i}(y)\right)\right): y \in L_{N}\right\}
$$

Then, $\Lambda$ has an equilibrium point in $K$.

Proof. For each $x \in X$, let $I(x)=\left\{i \in I: P_{i}(x) \neq \emptyset\right\}$. Define a mapping $P_{i}^{\prime}: X \rightarrow 2^{X}$ by $P_{i}^{\prime}(x)=$ $\pi_{i}^{-}\left(P_{i}(x)\right)=\Pi_{j \in I, j \neq i} X_{i} \otimes P_{i}(x)$ for each $x \in X$, where the mapping $\pi_{i}: X \rightarrow X_{i}$ is projection of $X$ onto $X_{i}$. Furthermore, define the mapping $P: X \rightarrow 2^{X}$ by

$$
P(x)= \begin{cases}\operatorname{Cif(x)}_{i} P_{i}^{\prime}(x) & \text { if } I(x) \neq \emptyset, \\ \emptyset & \text { if } I(x)=\emptyset .\end{cases}
$$

Then, for each $x \in X, P(x) \neq \emptyset$ if and only if $I(x) \neq \emptyset$. We will show that $P$ is an $\mathcal{A}_{C}$-majorized mapping. For each $x \in X$ with $P(x) \neq \emptyset$, let $i \in I(x)$ with $P_{i}(x) \neq \emptyset$, by (ii), and let $N_{x}$ be an open neighborhood of $x$ in $X$, and $\psi_{i, x}, \phi_{i, x}: X \rightarrow 2^{X_{i}}$ be mappings such that

(a) for each $z \in N_{x}, P_{i}(z) \subset \phi_{i, x}(z), z_{i} \notin \operatorname{co}_{\Gamma}\left(\phi_{i, x}(z)\right)$, and $\left\{z \in N_{x}: P_{i}(z) \neq \emptyset\right\} \subset\{z \in$ $\left.N_{x}: \psi_{i, x}(z) \neq \emptyset\right\}$,

(b) for each $z \in X, \psi_{i, x}(z) \subset \phi_{i, x}(z)$ and $\operatorname{co}_{\Gamma}\left(\phi_{i, x}(z)\right) \subset X_{i}$,

(c) the mapping $\psi_{i, x}^{-}: X_{i} \rightarrow 2^{X}$ is compactly open valued on $X_{i}$,

(d) for each finite subset $A \in\left\langle\operatorname{dom} P_{i}\right\rangle,\left\{z \in \cap_{x \in A} N_{x}: P_{i}(z) \neq \emptyset\right\} \subset\left\{z \in \cap_{x \in A} N_{x}\right.$ : $\left.\cap_{x \in A} \psi_{i, x}(z) \neq \emptyset\right\}$.

By (iii), we may assume that $N_{x} \subset W_{i}$, hence $P_{i}(z) \neq \emptyset$ and $i \in I(z)$ for all $z \in N_{x}$. Now, define two mappings $\psi_{x}, \phi_{x}: X \rightarrow 2^{X}$ by

$$
\psi_{x}(z)=\pi_{i}^{-}\left(\psi_{i, x}(z)\right), \quad \phi_{x}(z)=\pi_{i}^{-}\left(\phi_{i, x}(z)\right) \quad \text { for each } z \in X
$$

Then, we have

(a') for each $z \in N_{x}$, by (a), $P(z)=\cap_{i \in I(z)} P_{i}^{\prime}(z) \subset P_{i}^{\prime}(z)=\pi_{i}^{-}\left(P_{i}(z)\right) \subset \pi_{i}^{-}\left(\phi_{i, x}(z)\right)=$ $\phi_{x}(z)$ and $z \notin \operatorname{co}_{\Gamma}\left(\phi_{x}(z)\right)$,

$\left(\mathrm{b}^{\prime}\right)$ for each $z \in X, \psi_{x}(z) \subset \phi_{x}(z)$ by $(\mathrm{b})$,

$\left(c^{\prime}\right)$ for each $y \in X, \psi_{x}^{-}(y)=\left\{z \in X: y \in \psi_{x}(z)\right\}=\left\{z \in X: y_{i} \in \psi_{i, x}(z)\right\}=\psi_{i, x}^{-}(y)$ is compactly open in $X$ by $(\mathrm{c})$, 
(d') for each finite subset $A=\left\{x_{1}, x_{2}, \ldots, x_{n}\right\} \in\langle\operatorname{dom} P\rangle$, put $\cup\{B: B \subset A$ and $\left.\cap_{x \in B} I(x) \neq \emptyset\right\}=\left\{x_{i_{1}}, x_{i_{2}}, \ldots, x_{i_{m}}\right\}$. For each $i \in \cap_{k=1}^{m} I\left(x_{i_{k}}\right)$, then

$$
\bigcap_{x \in A} \psi_{x}(z)=\bigcap_{x \in A} \pi_{i}^{-}\left(\psi_{i, x}(z)\right)=\underset{j \neq i_{k}, k=1, \ldots, n}{\prod_{j}} X_{j} \stackrel{\bigcap}{m}_{k=1}^{m} \psi_{i, x_{i_{k}}}(z) \otimes \prod_{k=m+1}^{n} \psi_{i_{k}, x_{i_{k}}}(z) .
$$

For each $z \in \cap_{x \in A} N_{x}$, if $\cap_{x \in A} \psi_{x}(z)=\emptyset$, then there exists a set $A_{1}=\left\{x_{i_{1}}, x_{i_{2}}, \ldots, x_{i_{m}}\right\} \subset A$, such that $\cap_{x \in A_{1}} \psi_{i, x}(z)=\emptyset$, by (d), $P_{i}(z)=\emptyset$. Thus, $\left\{z \in \cap_{x \in A} N_{x}: P(z) \neq \emptyset\right\} \subset\left\{z \in \cap_{x \in A} N_{x}\right.$ : $\left.\cap_{x \in A} \psi_{x}(z) \neq \emptyset\right\}$. This shows that $P$ is an $\mathcal{A}_{C}$-majorized mapping. By $P^{-}(y)=P_{i}^{-}\left(\pi_{i}(y)\right)$ and condition (iv), for each $N \in\langle X\rangle$, there exists a compact abstract convex subset $L_{N}$ of $X$ containing $N$ such that

$$
L_{N} \backslash K \subset \bigcup\left\{\operatorname{cint}\left(P_{i}^{-}\left(\pi_{i}(y)\right)\right): y \in L_{N}\right\}=\bigcup\left\{\operatorname{cint}\left(P^{-}(y)\right): y \in L_{N}\right\} .
$$

Hence, all the hypotheses of Theorem 4.5 are satisfied. By Theorem 4.5, there exists a point $\widehat{x} \in K$ such that $P(\widehat{x})=\emptyset$. This implies that $I(\widehat{x})=\emptyset$ and therefore $P_{i}(\widehat{x})=\emptyset$ for each $i \in I$, that is, $\widehat{x}$ is an equilibrium point of $\Lambda$.

Remark 5.5. Theorem 5.4 improves and generalizes Theorem 5.2 of Ding and Feng [20], Theorem 6.2 of Ding and Wang [6], Theorem 4.2 of Yuan [25], Theorem 4.2 of Chowdhury et al. [4], and Theorem 4.2 of Tan and Yuan [26].

Applying Theorem 5.4, we prove that the following equilibria existence theorem for a noncompact generalized games.

Theorem 5.6. Let $\Lambda=\left(X ; X_{i} ; A_{i} ; B_{i} ; P_{i}, \pi\right)_{i \in I}$ be a generalized game. Let $K$ be a compact and closed subset of $X$ and $\operatorname{co}_{\Gamma}\left(\pi_{i}(K)\right)=\pi_{i}(K)$. Suppose that for each $i \in I$,

(i) $\left(X_{i}, \Gamma_{i}\right)$ is a paracompact abstract convex space such that $(X ; \Gamma)$ satisfies the partial KKM principle,

(ii) for each $x \in X, \operatorname{co}_{\Gamma}\left(\mathrm{A}_{\mathrm{i}}(\mathrm{x})\right) \subset \overline{\mathrm{B}}_{\mathrm{i}}(\mathrm{x}), A_{i}(x) \cap P_{i}(x) \subset \pi_{i}(K)$ and $\operatorname{dom} A_{i}=X$,

(iii) for each $y \in X_{i}, A_{i}^{-}(y)$ is compactly open in $X$,

(iv) $W_{i}=\left\{x \in X:\left(A_{i} \cap P_{i}\right)(x) \neq \emptyset\right\}$ is open in $X$,

(v) $A_{i} \cap P_{i}: X \rightarrow 2^{X_{i}}$ is an $A_{C}$-majorized mapping,

(vi) for each $N \in\langle X\rangle$, there exists a compact abstract convex subset $L_{N}$ of $X$ containing $N$ such that

$$
L_{N} \backslash K \subset \bigcup\left\{\operatorname{cint}\left(\left(A_{i} \cap P_{i}\right)^{-}(y)\right): y \in L_{N}\right\}
$$

Then, $\Lambda$ has an equilibrium point $\hat{x}$ in $K$. 
Proof. For each $i \in I$, let $F_{i}=\left\{x \in X: x_{i} \notin \bar{B}_{i}(x)\right\}$, then $F_{i}$ is open in $X$. Define $Q_{i}: X \rightarrow 2^{X_{i}}$ by

$$
Q_{i}(x)= \begin{cases}\pi_{i}(K) & \text { if } x \in X \backslash K \\ \left(A_{i} \cap P_{i}\right)(x) & \text { if } x \in K \backslash F_{i} \\ A_{i}(x) & \text { if } x \in F_{i} \cap K\end{cases}
$$

We will prove that the qualitative game $\Lambda^{\prime}=\left(X ; X_{i}, Q_{i}\right)_{i \in I}$ satisfies all the hypotheses of Theorem 5.4. For each $i \in I$, we have that the set

$$
\begin{aligned}
\left\{x \in X: Q_{i}(x) \neq \emptyset\right\} & =\left\{x \in X \backslash K: \pi_{i}(K) \neq \emptyset\right\} \cup\left\{x \in K \backslash F_{i}:\left(A_{i} \cap P_{i}\right)(x) \neq \emptyset\right\} \\
& \cup\left\{x \in K \cap F_{i}: A_{i}(x) \neq \emptyset\right\} \\
& =(X \backslash K) \cup\left[\left(K \backslash F_{i}\right) \cap W_{i}\right] \cup\left(K_{i} \cap F_{i}\right) \\
& =(X \backslash K) \cup\left[K \cap\left(W_{i} \cup F_{i}\right)\right] \\
& =(X \backslash K) \cup W_{i} \cup F_{i}
\end{aligned}
$$

is open in $X$ and hence the condition (iii) of Theorem 5.4 is satisfied. By (v), for each $x \in W_{i}$, there exist an open neighborhood $N_{x}$ of $x$ in $X$ and two mappings $\psi_{i, x}, \phi_{i, x}: X \rightarrow 2^{X_{i}}$ such that

(a) for each $z \in N_{x},\left(A_{i} \cap P_{i}\right)(z) \subset \phi_{i, x}(z), z_{i} \notin \operatorname{co}_{\Gamma}\left(\phi_{i, x}(z)\right)$ and $\left\{z \in N_{x}:\left(A_{i} \cap\right.\right.$ $\left.\left.P_{i}\right)(z) \neq \emptyset\right\} \subset\left\{z \in N_{x}: \psi_{i, x}(z) \neq \emptyset\right\}$,

(b) for each $z \in X, \psi_{i, x}(z) \subset \phi_{i, x}(z)$ and $\operatorname{co}_{\Gamma}\left(\phi_{i, x}(z)\right) \subset X_{i}$,

(c) the mapping $\psi_{i, x}^{-}: X_{i} \rightarrow 2^{X}$ is compactly open valued on $X_{i}$,

(d) for each nonempty finite subset $A \in\left\langle\operatorname{dom}\left(A_{i} \cap P_{i}\right)\right\rangle$,

$$
\left\{z \in \underset{x \in A}{\cap} N_{x}:\left(A_{i} \cap P_{i}\right)(z) \neq \emptyset\right\} \subset\left\{z \in \underset{x \in A}{\cap} N_{x}: \cap_{x \in A} \psi_{i, x}(z) \neq \emptyset\right\} .
$$

Define $\Psi_{i, x}, \Phi_{i, x}: X \rightarrow 2^{X_{i}}$ by

$$
\begin{gathered}
\Psi_{i, x}(z)= \begin{cases}\pi_{i}(K) & \text { if } z \in X \backslash K, \\
\psi_{i, x}(z) & \text { if } z \in K \backslash F_{i}, \\
A_{i}(z) & \text { if } z \in F_{i} \cap K,\end{cases} \\
\Phi_{i, x}(z)= \begin{cases}\pi_{i}(K) & \text { if } z \in X \backslash K, \\
\phi_{i, x}(z) & \text { if } z \in K \backslash F_{i}, \\
A_{i}(z) & \text { if } z \in F_{i} \cap K .\end{cases}
\end{gathered}
$$


Now for each $x \in X$ with $Q_{i}(x) \neq \emptyset$, the set

$$
U_{x}= \begin{cases}X \backslash K & \text { if } x \in X \backslash K, \\ N_{x} & \text { if } x \in K \backslash F_{i} \\ F_{i} & \text { if } x \in F_{i} \cap K\end{cases}
$$

is open in $X$. Then,

(a') for each $z \in U_{x}, Q_{i}(z) \subset \Phi_{i, x}(z)$ by (a) if $z \in X \backslash K, z_{i} \notin \operatorname{co}_{\Gamma}\left(\pi_{i}(K)\right)=\operatorname{co}_{\Gamma}\left(\Phi_{i, x}(z)\right)$ and if $z \in N_{x}, z \notin \operatorname{co}_{\Gamma}\left(\phi_{i, x}(z)\right)=\operatorname{co}_{\Gamma}\left(\Phi_{i, x}(z)\right)$ by (a) if $z \in F_{i}, z \notin \operatorname{co}_{\Gamma}\left(A_{i}(z)\right)=$ $\operatorname{co}_{\Gamma}\left(\Phi_{i, x}(z)\right)$ by (ii), that is, $z \notin \operatorname{co}_{\Gamma}\left(\Phi_{i, x}(z)\right)$ for each $z \in X$, and $\left\{z \in U_{x}\right.$ : $\left.Q_{i}(z) \neq \emptyset\right\} \subset\left\{z \in U_{x}: \Psi_{i, x}(z) \neq \emptyset\right\}$ by $(\mathrm{a})$,

(b') for each $z \in X, \Psi_{i, x}(z) \subset \Phi_{i, x}(z)$ by (b) and $\operatorname{co}_{\Gamma}\left(\Psi_{i, x}(z)\right) \subset X_{i}$,

(c') for each $y \in X_{i}$,

$$
\begin{aligned}
\Psi_{i, x}^{-}(y) & =\left\{z \in X: y \in \Psi_{i, x}(z)\right\}=\left\{z \in X \backslash K: y \in \pi_{i}(K)\right\} \\
& \cup\left\{z \in K \backslash F_{i}: y \in \psi_{i, x}(z)\right\} \cup\left\{z \in K \cap F_{i}: y \in A_{i}(z)\right\} \\
& =(X \backslash K) \cup\left[\psi_{i, x}^{-}(y) \cap\left(K \cap F_{i}\right)\right] \cup\left[A_{i}^{-}(y) \cap K \cap F_{i}\right]
\end{aligned}
$$

is compactly open by (c) and (iii),

(d') for each $A \in\left\langle\operatorname{dom} Q_{i}\right\rangle$, put

$$
A=[A \cap(X \backslash K)] \cup\left[A \cap\left(K \backslash F_{i}\right)\right] \cup\left[A \cap K \cap F_{i}\right]=A_{1} \cup A_{2} \cup A_{3} .
$$

Case I. If $A_{3} \neq \emptyset, \cap_{x \in A} U_{x} \subset F_{i} \cap K$, then

$$
\begin{aligned}
\left\{z \in \cap_{x \in A} U_{x}: \cap_{x \in A} \Psi_{i, x}(z) \neq \emptyset\right\} & =\left\{z \in \cap_{x \in A} U_{x}: A_{i}(z) \neq \emptyset\right\} \\
& =\left\{z \in \cap_{x \in A} U_{x}: Q_{i}(z) \neq \emptyset\right\} .
\end{aligned}
$$

Case II. If $A_{3}=\emptyset$, then

(1) if $A_{1} \neq \emptyset, A_{2} \neq \emptyset$, then $\cap_{x \in A} U_{x}=\left(\cap_{x \in A_{2}} N_{x}\right) \cap(X \backslash K) \subset X \backslash K$, and

$$
\begin{aligned}
\left\{z \in \cap_{x \in A} U_{x}: \underset{x \in A}{\cap} \Psi_{i, x}(z) \neq \emptyset\right\} & =\left\{z \in \underset{x \in A}{\cap} U_{x}: \pi_{i}(K) \neq \emptyset\right\} \\
& =\left\{z \in \cap_{x \in A} U_{x}: Q_{i}(z) \neq \emptyset\right\} .
\end{aligned}
$$


(2) If $A_{2}=\emptyset, A_{1} \neq \emptyset, \cap_{x \in A} U_{x}=X \backslash K$, that is similar to the condition (1).

(3) If $A_{1}=\emptyset, A_{2} \neq \emptyset$, then

$$
\begin{aligned}
& \left\{z \in \underset{x \in A}{\cap} U_{x}: \underset{x \in A}{\cap} \Psi_{i, x}(z) \neq \emptyset\right\}=\left\{z \in\left(\underset{x \in A_{2}}{\cap} N_{x}\right) \cap(X \backslash K): \Psi_{i, x}(z) \neq \emptyset\right\} \\
& \cup\left\{z \in\left(\underset{x \in A_{2}}{\cap} N_{x}\right) \cap\left(K \backslash F_{i}\right): \Psi_{i, x}(z) \neq \emptyset\right\} \\
& \cup\left\{z \in\left(\underset{x \in A_{2}}{\cap} N_{x}\right) \cap\left(K \cap F_{i}\right): \Psi_{i, x}(z) \neq \emptyset\right\} \\
& =\left\{z \in\left(\underset{x \in A_{2}}{\cap} N_{x}\right) \cap(X \backslash K): \pi_{i}(K) \neq \emptyset\right\} \\
& \cup\left\{z \in\left(\underset{x \in A_{2}}{\cap} N_{x}\right) \cap\left(K \backslash F_{i}\right): \underset{x \in A}{\cap} \psi_{i, x}(z) \neq \emptyset\right\} \\
& \cup\left\{z \in\left(\underset{x \in A_{2}}{\cap} N_{x}\right) \cap\left(K \cap F_{i}\right): A_{i}(z) \neq \emptyset\right\} \\
& \supset\left\{z \in\left(\underset{x \in A_{2}}{\cap} N_{x}\right) \cap(X \backslash K): \pi_{i}(K) \neq \emptyset\right\} \\
& \cup\left\{z \in\left(\underset{x \in A_{2}}{\cap} N_{x}\right) \cap\left(K \backslash F_{i}\right):\left(A_{i} \cap P_{i}\right)(z) \neq \emptyset\right\} \\
& \cup\left\{z \in\left(\underset{x \in A_{2}}{\cap} N_{x}\right) \cap\left(K \cap F_{i}\right): A_{i}(z) \neq \emptyset\right\} \\
& =\left\{z \in \underset{x \in A_{2}}{\cap} N_{x}: Q_{i}(z) \neq \emptyset\right\} \text {. }
\end{aligned}
$$

Thus, $Q_{i}$ is an $A_{C}$-majorized mapping. By the definition of $Q$ and condition (vi) for each $N \in\langle X\rangle$, there exists a compact abstract convex subset $L_{N}$ of $X$ containing $N$ such that

$$
L_{N} \backslash K \subset \bigcup\left\{\operatorname{cint}\left(\left(A_{i} \cap P_{i}\right)^{-}(y)\right): y \in L_{N}\right\} \subset \bigcup\left\{\operatorname{cint}\left(Q_{i}^{-}(y)\right): y \in L_{N}\right\} .
$$

Hence, all the hypotheses of Theorem 5.4 are satisfied. By Theorem 5.4, there exists a point $\widehat{x} \in K$ such that $Q_{i}(\widehat{x})=\emptyset(i \in I)$. By the definition of $Q_{i}, \widehat{x} \in K \backslash F_{i} \subset K$, that is for all $i \in I$, $\widehat{x}_{i} \in \bar{B}_{i}(\widehat{x})$, and $A_{i}(\widehat{x}) \cap P_{i}(\widehat{x})=\emptyset$. This completes the proof.

Remark 5.7. Theorem 5.6 generalized Theorem 5.3 of Ding and Feng [20], Theorem 6.3 of Ding and Wang [6], Theorem 4.4 of Chowdhury et al. [4], and Theorem 4.3 of Tan and Yuan [26].

\section{Acknowledgment}

This work is partially supported by the National Youth Fund of China (51203112). 


\section{References}

[1] A. Borglin and H. Keiding, "Existence of equilibrium actions and of equilibrium: a note on the "new" existence theorems," Journal of Mathematical Economics, vol. 3, no. 3, pp. 313-316, 1976.

[2] X. P. Ding, "Coincidence theorems and equilibria of generalized games," Indian Journal of Pure and Applied Mathematics, vol. 27, no. 11, pp. 1057-1071, 1996.

[3] Z.-F. Shen, "Maximal element theorems of $H$-majorized correspondence and existence of equilibrium for abstract economies," Journal of Mathematical Analysis and Applications, vol. 256, no. 1, pp. 67-79, 2001.

[4] M. S. R. Chowdhury, E. Tarafdar, and K.-K. Tan, "Minimax inequalities on G-convex spaces with applications to generalized games," Nonlinear Analysis. Theory, Methods E Applications A, vol. 43, no. 2, pp. 253-275, 2001.

[5] W. Briec and C. Horvath, "Nash points, Ky Fan inequality and equilibria of abstract economies in Max-Plus and B-convexity," Journal of Mathematical Analysis and Applications, vol. 341, no. 1, pp. 188199, 2008.

[6] X. P. Ding and L. Wang, "Fixed points, minimax inequalities and equilibria of noncompact abstract economies in FC-spaces," Nonlinear Analysis. Theory, Methods \& Applications A, vol. 69, no. 2, pp. 730746, 2008.

[7] W. K. Kim, S. Kum, and K. H. Lee, "On general best proximity pairs and equilibrium pairs in free abstract economies," Nonlinear Analysis. Theory, Methods \& Applications A, vol. 68, no. 8, pp. 22162227, 2008.

[8] W. K. Kim and K. K. Tan, "New existence theorems of equilibria and applications," Nonlinear Analysis, vol. 47, pp. 531-542, 2001.

[9] L.-J. Lin, L.-F. Chen, and Q. H. Ansari, "Generalized abstract economy and systems of generalized vector quasi-equilibrium problems," Journal of Computational and Applied Mathematics, vol. 208, no. 2, pp. 341-353, 2007.

[10] L. J. Lin and Y. H. Liu, "The study of abstract economies with two constraint correspondences," Journal of Optimization Theory and Applications, vol. 137, no. 1, pp. 41-52, 2008.

[11] Y.-M. Du and L. Deng, "Equilibrium existence theorems of generalized games for generalized $\mathcal{L}_{\theta, F_{c}}{ }^{-}$ majorized mapping in topological space," Nonlinear Analysis. Theory, Methods \& Applications A, vol. 67, no. 1, pp. 316-326, 2007.

[12] C. D. Horvath, "Contractibility and generalized convexity," Journal of Mathematical Analysis and Applications, vol. 156, no. 2, pp. 341-357, 1991.

[13] C. Horvath, Nonlinear and Convex Analysis, Marcel Dekker, NewYork, NY, USA, 1987.

[14] S. Park and H. Kim, "Admissible classes of multifunctions on generalized convex spaces," Proceedings of College of Natural Sciences, Seoul National University, vol. 18, pp. 1-21, 1993.

[15] X. P. Ding, "Maximal element theorems in product FC-spaces and generalized games," Journal of Mathematical Analysis and Applications, vol. 305, no. 1, pp. 29-42, 2005.

[16] J.-C. Hou, "An existence theorem of equilibrium for generalized games in $H$-spaces," Applied Mathematics Letters, vol. 16, no. 1, pp. 97-103, 2003.

[17] X. P. Ding, "Maximal elements for $G_{B}$-majorized mappings in product $G$-convex spaces and applications. I," Applied Mathematics and Mechanics, vol. 24, no. 6, pp. 583-594, 2003.

[18] X. P. Ding and F. Q. Xia, "Equilibria of nonparacompact generalized games with $\mathcal{L}_{F_{c}}$-majorized correspondence in G-convex spaces," Nonlinear Analysis. Theory, Methods \& Applications A, vol. 56, no. 6 , pp. 831-849, 2004.

[19] M.-G. Yang and L. Deng, "Equilibria of nonparacompact generalized games with $\mathcal{L}_{c}$-majorized correspondences in FC-spaces," Nonlinear Analysis. Theory, Methods $\mathcal{E}$ Applications A, vol. 70, no. 2, pp. 890-903, 2009.

[20] X. P. Ding and H. R. Feng, "Fixed point theorems and existence of equilibrium points of noncompact abstract economies for $\mathcal{L}_{F}$-majorized mappings in FC-spaces," Nonlinear Analysis. Theory, Methods $\mathcal{E}$ Applications A, vol. 72, no. 1, pp. 65-76, 2010.

[21] S. Park, "On generalizations of the KKM principle on abstract convex spaces," Nonlinear Analysis Forum. An International Journal, vol. 11, no. 1, pp. 67-77, 2006.

[22] S. Park, "The KKM principle in abstract convex spaces: equivalent formulations and applications," Nonlinear Analysis. Theory, Methods E Applications A, vol. 73, no. 4, pp. 1028-1042, 2010.

[23] S. Park, "Elements of the KKM theory on abstract convex spaces," Journal of the Korean Mathematical Society, vol. 45, no. 1, pp. 1-27, 2008. 
[24] S. Park, "New generalizations of basic theorems in the KKM theory," Nonlinear Analysis. Theory, Methods \& Applications A, vol. 74, no. 9, pp. 3000-3010, 2011.

[25] G. X.-Z. Yuan, "The study of equilibria for abstract economics in topological vector spaces-a unified approach," Nonlinear Analysis. Theory, Methods \& Applications A, vol. 37, no. 4, pp. 409-430, 1999.

[26] K.-K. Tan and Z.-Z. Yuan, "A minimax inequality with applications to existence of equilibrium points," Bulletin of the Australian Mathematical Society, vol. 47, no. 3, pp. 483-503, 1993.

[27] N. C. Yannelis, "Maximal elements over noncompact subsets of linear topological spaces," Economics Letters, vol. 17, no. 1-2, pp. 133-136, 1985.

[28] X. P. Ding, "New H-KKM theorems and their applications to geometric property, coincidence theorems, minimax inequality and maximal elements," Indian Journal of Pure and Applied Mathematics, vol. 26, no. 1, pp. 1-19, 1995.

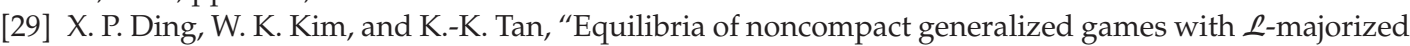
preference correspondences," Journal of Mathematical Analysis and Applications, vol. 164, no. 2, pp. 508 517, 1992.

[30] X. Ding, "Fixed points, minimax inequalities and equilibria of noncompact abstract economies," Taiwanese Journal of Mathematics, vol. 2, no. 1, pp. 25-55, 1998.

[31] J. Dugundji, Topology, Allyn and Bacon, Boston, Mass, USA, 1966.

[32] D. Gale and A. Mas-Colell, "On the role of complete, transitive preferences in equilibrium theory," in Equilibrium and Disequilibrium in Economic Theory, G. Schwodiauer, Ed., pp. 7-14, D. Reidel, Dordrecht, The Netherland, 1978.

[33] S. Park, "The Fan minimax inequality implies the Nash equilibrium theorem," Applied Mathematics Letters, vol. 24, no. 12, pp. 2206-2210, 2011. 


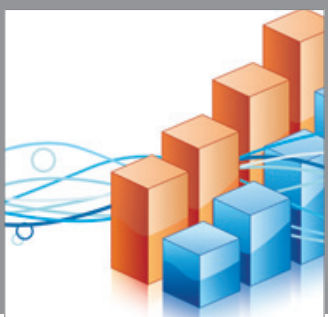

Advances in

Operations Research

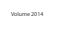

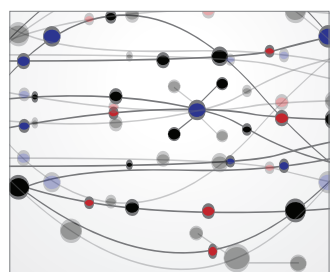

\section{The Scientific} World Journal
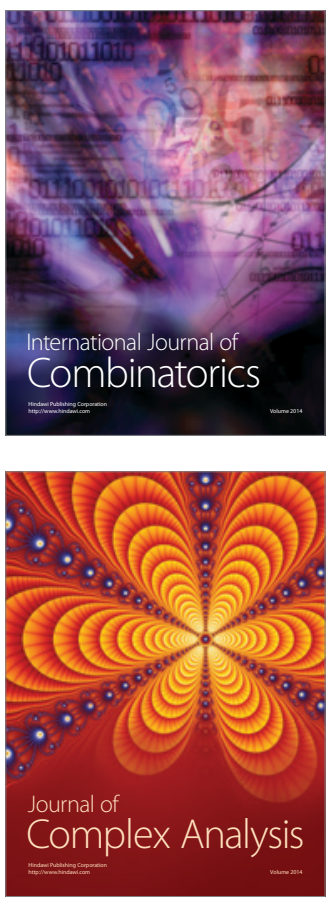

International Journal of

Mathematics and

Mathematical

Sciences
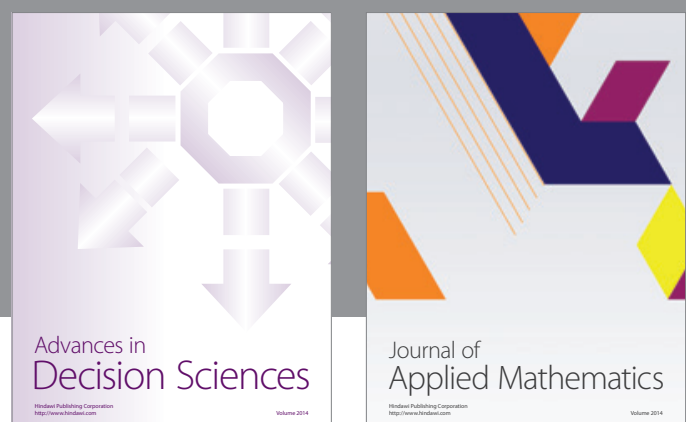

Journal of

Applied Mathematics
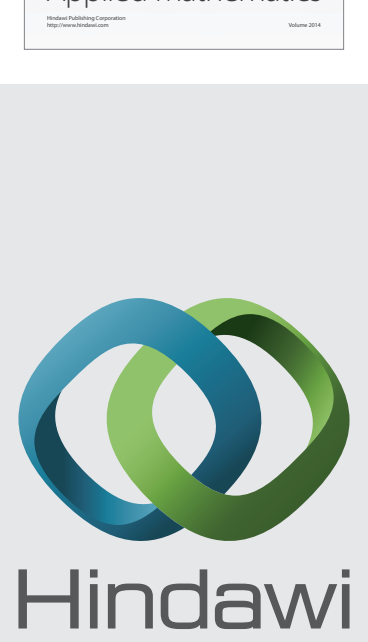

Submit your manuscripts at http://www.hindawi.com
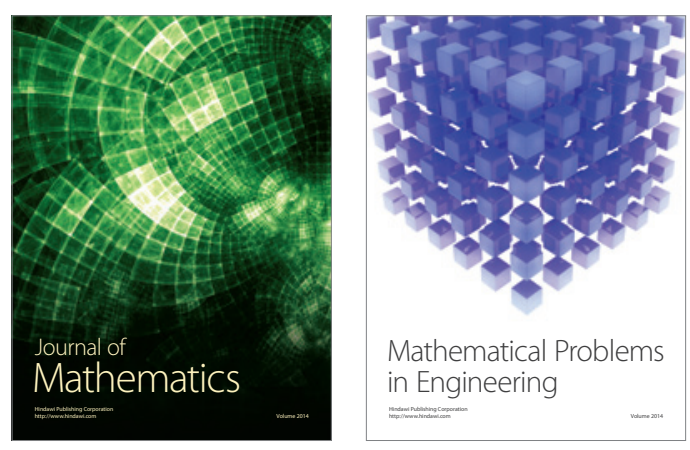

Mathematical Problems in Engineering
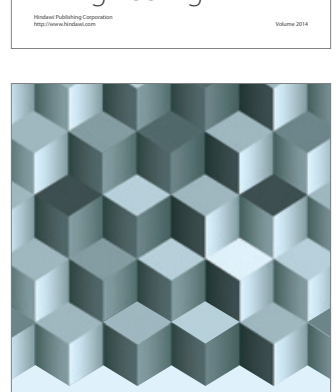

Journal of

Function Spaces
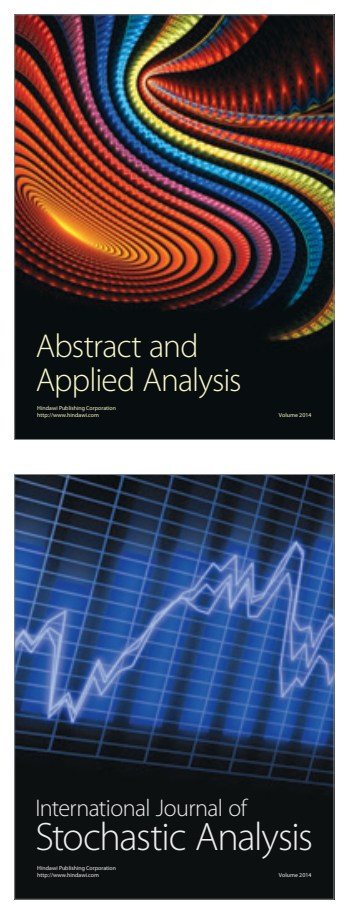

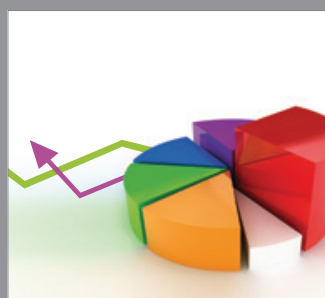

ournal of

Probability and Statistics

Promensencen
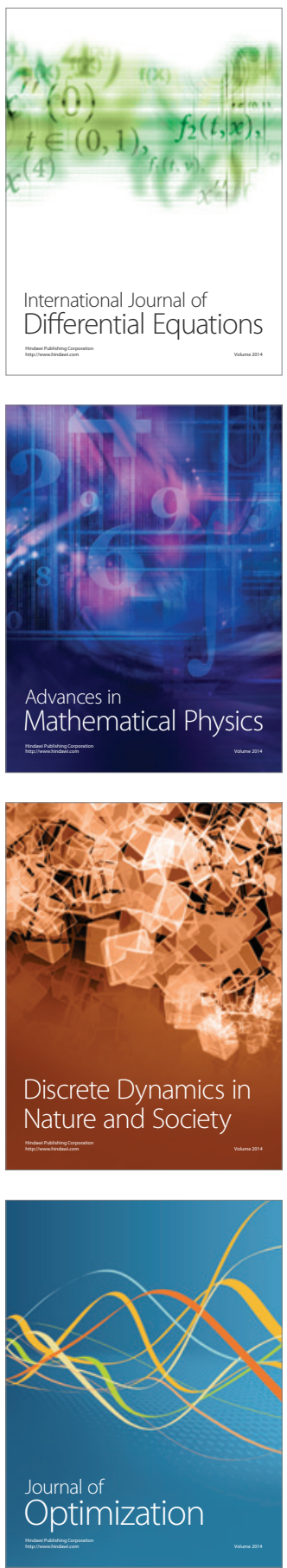